\title{
QUADERNI Quaderni
}

Communication, technologies, pouvoir

82 | Automne 2013

L'hôpital à l'épreuve de la performance économique

\section{Manuel Castells, Communication et pouvoir}

Jean-Christophe Plantin

\section{CpenEdition}

Journals

Édition électronique

URL : https://journals.openedition.org/quaderni/753

DOI : $10.4000 /$ quaderni.753

ISSN : 2105-2956

Éditeur

Les éditions de la Maison des sciences de l'Homme

Édition imprimée

Date de publication : 5 octobre 2013

Pagination : 109-111

Référence électronique

Jean-Christophe Plantin, «Manuel Castells,Communication et pouvoir », Quaderni [En ligne], 82 |

Automne 2013, mis en ligne le 05 octobre 2015, consulté le 08 mars 2022. URL : http://

journals.openedition.org/quaderni/753; DOI : https://doi.org/10.4000/quaderni.753 


\section{Compte rendu}

\section{Communication et pouvoir Manuel Castells}

Éditions de la MSH, Paris, 2013

1. Jacques Ellul, I'homme entier, film de Serge Steyer, 1993, $<$ http://www.elluldvd.com/> par Jean-Christophe Plantin Laboratoire COSTECH Université de Technologie de Compiègne

À la fin de sa vie, Jacques Ellul a affirmé ne pas avoir écrit une cinquantaine de livres, mais plutôt un seul livre qu'il a décliné en une cinquantaine de chapitres ${ }^{1}$. Communication et pouvoir constitue un nouveau chapitre du grand livre que le sociologue espagnol Manuel Castells écrit depuis plus de quarante ans sur les conséquences sociales et urbaines des TIC. Publié en 2009 en anglais et traduit en 2013 par les éditions de la MSH, ce dernier opus complète plus particulièrement ses travaux menés tout au long de sa trilogie sur « l'ère de l'information », pour proposer une grille de lecture des sociétés actuelles : au vu des formes réticulaires que prend l'organisation du pouvoir à l'heure de la mondialisation, et à partir du rôle des technologies de communication dans cette configuration, est-il pertinent de décrire les sociétés comme " post-modernes »? Sommes nous au contraire toujours dans la modernité, ou avons-nous atteint, comme Alain Touraine le suggère dans sa préface à l'ouvrage, une ère post-industrielle?

M. Castells se propose de répondre à cette question en affirmant que nous vivons dans l'ère de la communication. Pour le prouver, il analyse le rôle des TIC dans la construction et la mutation des rapports de pouvoir, en avançant l'hypothèse que le pouvoir repose dans nos sociétés sur le contrôle des moyens d'information et de communication. L'argumentation de son livre suit donc le déroulement suivant : l'État s'insère dans des réseaux mondialisés au sein desquels le pouvoir de groupes sociaux sur d'autres est relié à la capacité de contrôler ces réseaux. Cette dernière prend deux formes : la programmation/ reprogrammation des réseaux, qui désigne la possibilité de créer un réseau et d'agir sur sa finalité ; la commutation, qui connecte et assure la coopération 
entre plusieurs réseaux. Si la première capacité permet le contrôle de la communication, la seconde gère la connexion entre plusieurs réseaux, par exemple entre politique et médias. Manuel Castells prend comme exemple de reprogrammation des médias et de commutation entre réseaux le thème du réchauffement climatique. Dans ce qu'il appelle « the greening of media », et en suivant une approche voisine de la sociologie des problèmes publics, il montre comment ce débat est passé des mains des scientifiques à celles d'organisations environnementales, qui sont depuis devenues une source de référence également importante pour les décideurs et les médias.

Les concepts de re/programmation et de commutation sont particulièrement pertinents, car ils soulignent le fait que la résistance aux réseaux passe par les mêmes armes dont disposent ceux qui les contrôlent. Afin de prouver cela, M. Castells adopte une approche multiscalaire, prolongeant les théoriciens des sciences de la communication pour faire tenir ensemble l'analyse des médias de masse, des réseaux horizontaux et de ce qu'il nomme " l'autocommunication de masse ». L'auto-communication de masse définit les formes de communication en ligne s'appuyant sur le soi (« self ») tout en prenant place dans un réseau mondialisé. Pour caractériser l'autocommunication de masse, il nous affirme que « son contenu est autogénéré, son émission autodirigée et sa réception autodirigée par la masse de ceux qui communiquent avec la masse des autres» (p. 110). En ce sens, ce modèle comprend à la fois communication interpersonnelle, communication de masse et auto-communication de masse. Par exemple, Twitter permet de discuter avec un proche, de diffuser un message à plusieurs personnes, tout en s'appuyant par ailleurs sur les goûts et les opinions personnelles et participant à la construction de soi.

Manuel Castells assigne plusieurs objectifs à cette notion d'autocommunication de masse. Tout d'abord, elle lui permet de mettre l'individu au centre dans ces multiples enjeux de pouvoir, compensant la grille d'analyse parfois trop macro adoptée dans ses travaux précédents. L'attention portée ici à l'économie cognitive des individus constitue alors un apport judicieux pour compléter l'approche par les réseaux, tout en désamorçant la métaphore facile entre réseaux de communication et réseaux individuels neuronaux. Ensuite, cette notion permet de faire le lien avec les formes précédentes de communication également supports de pratiques expressives, tels que les radios pirates et autres médias alternatifs ${ }^{2}$. La nouveauté est que cette

2. Olivier Blondeau, Laurence Allard, Devenir Média, L'activisme sur Internet entre défection et expérimentation, Paris, Éditions Amsterdam, 2007. 
3. Henry Jenkins, Convergence Culture: Where Old and New Media Collide, New York, NYU Press, 2006.
4. Noortje Marres, « Testing Powers of Engagement Green Living Experiments, the Ontological Turn and the Undoability of Involvement », European Journal of Social Theory, vol. 12, 1, 2009, pp. 117-133. forme de communication se décline aujourd'hui à la fois sur le web, dans les jeux vidéos, ou encore au sein d'univers virtuels en ligne. De plus, à la suite de Jenkins ${ }^{3}$, M. Castells ne décrit pas cette auto-communication de masse uniquement comme une libération des individus : les médias en ligne restent intrinsèquement reliés à des intérêts commerciaux, dont le mode de fonctionnement de ce secteur constitue d'ailleurs un exemple canonique de réseau mondialisé, dont il analyse les différents hubs. Enfin, cette autocommunication de masse présente de nouvelles opportunités politiques, et peut être la base de stratégies de campagnes électorales, comme le décrit M. Castells à travers son analyse de celle d'Obama en 2008. Toutefois, cette communication à partir du soi crée également une culture de l'autopromotion des candidats, avec le rôle important du scandale comme stratégie politique, fait que Castells illustre à travers sa généalogie de la " politique du scandale » en Espagne depuis les années 90.

En conclusion, cet ouvrage permet de rappeler les qualités pédagogiques et empiriques de Manuel Castells : il expose en effet son propos pas à pas, en définissant au fur et à mesure les termes qu'il mobilise et en accompagnant le lecteur à grand renfort de citations. Ce style permet ainsi à Manuel Castells de déployer son cadre théorique sur une multitude de terrains, liant un grand effort de documentation avec des ressources quantitatives, le tout en effectuant un constant aller-retour entre le local et le global, des individus aux réseaux. On peut toutefois regretter que Manuel Castells cantonne son analyse aux lieux traditionnels du pouvoir, tels que les institutions ou durant des élections présidentielles, déjà abondamment analysés. On aurait par exemple apprécié connaître son analyse des formes d'engagement et de participation de publics à des causes politiques en dehors des cadres traditionnels d'analyse, en écho par exemple à ce que Noortje Marres propose de faire lorsqu'elle étudie la naissance d'une conscience écologique à travers la médiation des compteurs verts domestiques ${ }^{4}$. De même, une typologie des réseaux en fonction des difficultés de leur reprogrammation aurait également permis de gagner en puissance d'analyse. Il est par exemple possible, à la suite de l'action d'un « lanceur d'alerte » particulier (ici un insider « repenti »), d'avoir accès aux informations concernant le programme de surveillance américain PRISM. Toutefois, ce réseau reste très difficile à reprogrammer. 
